\title{
LIKELY PATH TO EXTINCTION IN SIMPLE BRANCHING MODELS WITH LARGE INITIAL POPULATION
}

\author{
F. C. KLEBANER AND R. LIPTSER
}

Received 15 September 2005; Revised 24 November 2005; Accepted 4 December 2005

We give explicit formulae for most likely paths to extinction in simple branching models when initial population is large. In discrete time, we study the Galton-Watson process, and in continuous time, the branching diffusion. The most likely paths are found with the help of the large deviation principle (LDP). We also find asymptotics for the extinction probability, which gives a new expression in continuous time and recovers the known formula in discrete time. Due to the nonnegativity of the processes, the proof of LDP at the point of extinction uses a nonstandard argument of independent interest.

Copyright (c) 2006 F. C. Klebaner and R. Liptser. This is an open access article distributed under the Creative Commons Attribution License, which permits unrestricted use, distribution, and reproduction in any medium, provided the original work is properly cited.

\section{Introduction and main results}

In population genetics it is often important to look back at the development of populations. In this paper we consider the question of how extinctions occur, and in particular, what path a population takes on the road to extinction. Using asymptotic analysis when initial population values are large, we are able to find the most likely path to extinction as well as the extinction probability in two simple branching models in discrete and continuous time. In both examples we use the large deviation principle (LDP) which is nonstandard since random processes are nonnegative, and we use trajectories ending up at zero.

One of the contributions of this paper is in rigorous proofs of the LDP for processes on half space. It may appear to the reader that the LDP follows from known results in Markov chains and diffusions. This is only partly correct. The standard proof of the lower bound in the local LDP relies on the change of measure. This requires a certain point (the point where maximum in the Fenchel-Legendre transform is achieved) to be finite. In our case this point is at infinity, breaking down the standard approach. We therefore give complete proofs of LDP's in Section 4 (discrete time) and Section 5 (continuous time) following the scheme of Puhalskii [19]. His approach states that the LDP is equivalent to exponential tightness plus local LDP, and is based on the stochastic exponential method(rather 
than the Laplace transform). Although we follow the scheme of Puhalskii [19] we do not use idempotent probability and give direct proofs. Since these proofs are more technical, we placed them at the end, after results on extinction. Once the LDP is established, the problem of finding the most likely path to extinction is in effect the problem of minimization of the rate function. This is typically a difficult problem due to nonlinearity. We are able to solve it by setting up the Bellman equation in discrete case, Section 2, and a dynamical control problem in continuous case, Section 3.

1.1. Galton-Watson process. A prototype of a branching model in discrete time is the Galton-Watson process, described as follows.

Let $X_{n}$ denote the population size at time $n$, and $\xi_{n+1}^{j}$ the number of offspring of the $j$ th individual. For each $n=1,2, \ldots,\left\{\left(\xi_{n}^{j}\right)_{j \geq 1}\right\}$ is the sequence of independent identically distributed integer-valued random variables with the probability distribution function $P\left(\xi_{n}^{j}=\ell\right)=p_{\ell}, \ell=0,1, \ldots$ The population size at time $n+1$ is given by

$$
X_{n+1}=\sum_{j=1}^{X_{n}} \xi_{n+1}^{j},
$$

where $X_{0}=K>0$. The state $\{0\}$ is absorbing, and the branching process $\left(X_{n}\right)_{n \geq 0}$ might be absorbed in $\{0\}$ at the extinction time

$$
\tau=\inf \left\{n: X_{n}=0\right\}
$$

If $p_{0}=0$, the population does not become extinct. However if $p_{0}>0$, it is well known (see, e.g., $[2,7])$ that the extinction time $\tau$ is finite with probability one if and only if the offspring mean $\mathfrak{m}=\sum_{\ell \geq 1} \ell_{p_{\ell}}$ does not exceed one $(\mathfrak{m} \leq 1)$. Moreover, for any $\mathfrak{m}$, the distribution function of $\tau$ is computed using the offspring probability generating function $\mathfrak{f}(s)=\sum_{\ell \geq 0} p_{\ell} s^{\ell}, 0 \leq s \leq 1$ : for any $N \geq 1$,

$$
P(\tau \leq N)=\left(\mathfrak{f}_{N}(0)\right)^{K},
$$

where $\mathfrak{f}_{n}(s)$ is the $n$th iterate of $\mathfrak{f}(s)$, that is, $\mathfrak{f}_{n}(s)=\mathfrak{f}\left(\mathfrak{f}_{n-1}(s)\right)$ with $\mathfrak{f}_{1}(0)=\mathfrak{f}(0)=p_{0}$.

A natural question is how to find the "path to extinction" given that extinction occurred at time $N, \tau=N$. The conditional distribution of the chain conditioned on extinction: for $n=1, \ldots, N-1$,

$$
\pi_{n \mid N}(i):=P\left(X_{n}=i \mid \tau=N\right), \quad i=1,2, \ldots,
$$

gives the complete description. It can be used to find the conditional median or the traditional optimal in the mean-square sense estimate $\hat{X}_{n}=\sum_{i=1}^{\infty} i \pi_{n \mid N}(i)$. Unfortunately such computations are involved, even using the Markov property of $\left(X_{n}\right)$. However, for large values of $X_{0}=K$, one path has an overwhelmingly large probability compared to the rest. Consider the normed branching process

$$
x_{n}^{K}=\frac{X_{n}}{K} .
$$


The limit in probability $P$ - $\lim _{K \rightarrow \infty} x_{n}^{K}=\hat{x}_{n}$ exists (see $\left.[9,13]\right)$ and satisfies $\hat{x}_{n+1}=\mathfrak{m} \hat{x}_{n}$, $\hat{x}_{0}=1$. The process $\hat{x}_{n}$ is always positive, irrespective of the value of $\mathfrak{m}$, so that the approximation $\hat{x}_{n}$ is inadequate for the study of extinction, the fact is already mentioned in [3]. In the approach we take that $\left(x_{n}^{K}\right)_{n \leq N}$ is approximated on the set $\{\tau \leq N\}$ by a deterministic sequence $u_{\text {. }}^{*}:=\left(u_{n}^{*}\right)_{n \leq N}$ with $u_{0}^{*}=1$, positive $u_{n}^{*}$ 's, and $u_{N}^{*}=0$, such that for small $\delta>0$ and large $K$,

$$
P\left(\sum_{n=1}^{N}\left|x_{n}^{K}-u_{n}^{*}\right| \leq \delta\right) \approx P(\tau \leq N) .
$$

This choice of $u^{*}$ might be warranted by the following argument. Since $\mathfrak{f}_{n}(0)$ increases in $n$, for large $K,\left(\mathfrak{f}_{N}(0)\right)^{K}$ is considerably larger than any of $\left(\mathfrak{f}_{n}(0)\right)^{K}$ for $n<N$. Then, by (1.3), $P(\tau \leq N)=P(\tau=N)+P(\tau \leq N-1) \approx P(\tau=N)$. Consequently, for any $u$. $=$ $\left(u_{n}\right)_{n \leq N}$ with $u_{0}=1$ and $u_{n} \geq 0$,

$$
P\left(\sum_{n=1}^{N}\left|x_{n}^{K}-u_{n}\right| \leq \delta\right) \lesssim P(\tau \leq N)
$$

For large $K$, extinction for the process $x_{n}^{K}$ is a rare event, since the limit process $\hat{x}_{n}$ is positive. Therefore, as in [12], we approach the problem of extinction using the large deviations theory, obtaining a new result as well as recovering an asymptotic version of the well-known result (1.3) by using this theory. According to LDP and Theorem 4.1, and by analogy with the maximal likelihood estimator, the path $\left(u_{n}^{*}\right)_{n \leq N}$ is said to be the most likely path to extinction of the normed population $x_{n}^{K}$.

Clearly, $\tau$ is the extinction time for both processes $X_{n}$ and $x_{n}^{K}$, so that $K u_{n}^{*}$ (with large $K)$ sets the pattern for the extinction path in the original branching process.

Figure 1.1 demonstrates likely paths to extinction for a binary splitting model with different parameters, $p=p_{0}$, illustrating the general result.

For formulating the main result, we use the log moment generating function, assuming its existence up to some $t_{0}>0$ :

$$
\mathfrak{g}(t)=\log \sum_{\ell \geq 0} e^{t \ell} p_{\ell}, \quad t \in\left(-\infty, t_{0}\right)
$$

It is related to the moment generating function by

$$
\log \mathfrak{f}_{n}(0) \equiv \mathfrak{g}_{n}(-\infty) \quad(\text { Lemma 2.1) }
$$

Theorem 1.1. Assume $p_{0}>0$ and (1.8). Then, for any $N \geq 1$,

(i)

$$
\left(u_{n}^{*}\right)_{n \leq N}=\underset{\substack{u_{0}=1, u_{N}=0 \\ u_{n}>0, n \leq N-1}}{\operatorname{argmax}} \lim _{\delta \rightarrow 0} \lim _{K \rightarrow \infty} \frac{1}{K} \log P\left(\sum_{n=1}^{N}\left|x_{n}^{K}-u_{n}\right| \leq \delta\right)
$$


4 Path to extinction in branching models

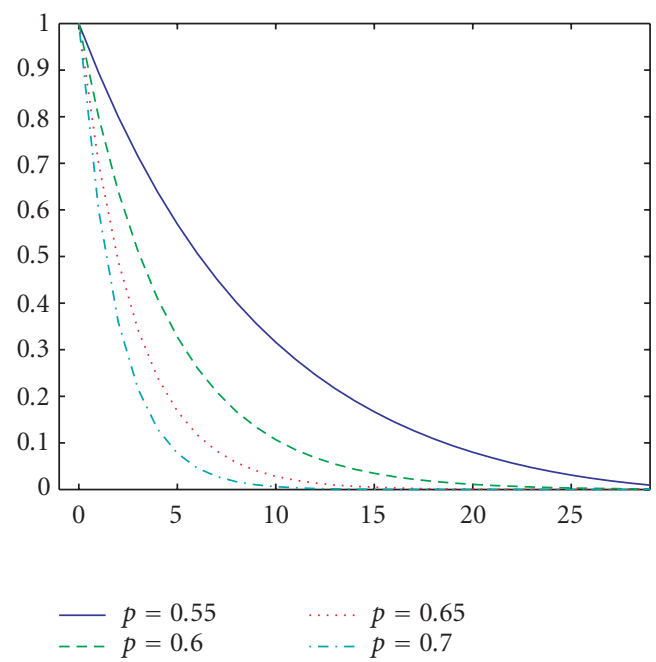

Figure 1.1

is given by

$$
u_{n}^{*}=\prod_{1 \leq i \leq n} \mathfrak{g}^{\prime}\left(\mathfrak{g}_{N-i}(-\infty)\right), \quad n \leq N
$$

where $\mathfrak{g}_{i}(t)$ is the ith iterate of $\mathfrak{g}(t), \mathfrak{g}_{0}(t)=t$,

(ii)

$$
\lim _{\delta \rightarrow 0} \lim _{K \rightarrow \infty} \frac{1}{K} \log P\left(\sum_{n=1}^{N}\left|x_{n}^{K}-u_{n}^{*}\right| \leq \delta\right)=\lim _{K \rightarrow \infty} \frac{1}{K} \log P(\tau \leq n) .
$$

1.2. Branching diffusion. In continuous time, we consider the model of a branching diffusion $X_{t}$ defined by the Itô equation

$$
d X_{t}=\alpha X_{t} d t+\sigma \sqrt{X_{t}} d B_{t}
$$

with a positive initial condition $X_{0}=K$, where $B_{t}$ is a Brownian motion, $\sigma^{2}>0$, and $\alpha \in$ $\mathbb{R}$. Stochastic equation(1.13) possesses a strong nonnegative solution. Since the diffusion parameter degenerates, one way to see this is to construct the solution from the following approximating sequence $\left(X_{t}^{i}\right)_{i \geq 1}$ :

$$
X_{t}:=X_{t}^{1} I_{\left\{t \leq \tau_{1}\right\}}+\sum_{i \geq 1} X_{\tau_{i}}^{i} I_{\left\{\tau_{i}<t \leq \tau_{i+1}\right\}}
$$

where $d X_{t}^{i}=\alpha X_{t}^{i} d t+\sigma \sqrt{\left|X_{t}^{i}\right| \vee i^{-1}} d B_{t}, X_{0}^{i}=K$, and $\tau_{i}=\inf \left\{X_{t}^{i} \leq i^{-1}\right\}$ the increasing sequence of stopping times $\left(\tau_{i}\right)_{i \geq 1}$ relative to the filtration generated by the Brownian motion $\left(B_{t}\right)$ (see also [10, Theorem 13.1]). The strong uniqueness of (1.13) follows from Yamada-Watanabe's theorem (see, e.g., Rogers and Williams [21, page 265]) since its drift 
and diffusion parameters are Lipschitz- and Hölder- (with coefficient 1/2) continuous, respectively.

Obviously,

$$
\tau=\inf \left\{t: X_{t}=0\right\}=\lim _{i \rightarrow \infty} \tau_{i}
$$

We analyze the normed process $x_{t}^{K}=X_{t} / K$. Due to (1.13), $x_{t}^{K}$ solves the Itô equation

$$
d x_{t}^{K}=\alpha x_{t}^{K} d t+\frac{\sigma}{\sqrt{K}} \sqrt{x_{t}^{K}} d B_{t}
$$

with $x_{0}^{K}=1$. It can be readily shown that $P$ - $\lim _{K \rightarrow \infty} x_{t}^{K}=\hat{x}_{t}$ exists and solves $d \hat{x}_{t} / d t=$ $\alpha \hat{x}_{t}, \hat{x}_{0}=1$. However, $\hat{x}_{t}$ is always positive and is far from being an estimated path to extinction. As in the discrete time, in order to evaluate the path to extinction for $\left(x_{t}^{K}\right)_{t \leq T}$ for fixed $T>0$, we approximate $\left(x_{t}^{K}\right)_{t \leq T}$ on the set $\{\tau \leq T\}$ by a deterministic function $\left(u_{t}^{*}\right)_{t \leq T}$ with $u_{0}^{*}=1, u_{T}^{*}=0$, and $u_{t}^{*}>0$, such that for a small $\delta>0$ and large $K$,

$$
P\left(\sup _{t \leq T}\left|x_{t}^{K}-u_{t}^{*}\right| \leq \delta\right) \geq P\left(\sup _{t \leq T}\left|x_{t}^{K}-u_{t}\right| \leq \delta\right)
$$

for any $\left(u_{t}\right)_{t \leq T}$ from the set $\left\{u_{0}=1,\left(u_{t}>0\right)_{t<T}, u_{T}=0\right\}$.

Unfortunately, the useful formula of (1.3) type is not known to us in this case. Here we obtain its asymptotic version as $K \rightarrow \infty$, see (ii) below.

Theorem 1.2. For any $T>0$,

(i)

$$
\left(u_{t}^{*}\right)_{t \leq T}=\underset{\substack{u_{0}=1, u_{T}=0 \\ u_{t}>0, t<T}}{\operatorname{argmax}} \lim _{\delta \rightarrow 0} \lim _{K \rightarrow \infty} \frac{1}{K} \log P\left(\sup _{t \leq T}\left|x_{t}^{K}-u_{t}\right| \leq \delta\right)
$$

is given by

$$
u_{t}^{*}= \begin{cases}e^{-\alpha t}\left(1-\frac{1-e^{-\alpha t}}{1-e^{-\alpha T}}\right)^{2}, & \alpha \neq 0, \\ \left(1-\frac{t}{T}\right)^{2}, & \alpha=0\end{cases}
$$

(ii)

$$
\begin{aligned}
\lim _{K \rightarrow \infty} \frac{1}{K} \log P(\tau \leq T) & =\lim _{\delta \rightarrow 0} \lim _{K \rightarrow \infty} \frac{1}{K} \log P\left(\sup _{t \leq T}\left|x_{t}^{K}-u_{t}^{*}\right| \leq \delta\right) \\
& =- \begin{cases}\frac{1}{\sigma^{2}} \frac{\alpha}{1-e^{-\alpha T}}, & \alpha \neq 0, \\
\frac{1}{\sigma^{2} T}, & \alpha=0 .\end{cases}
\end{aligned}
$$


Corollary 1.3. (1) $u^{*}$. has the remarkable property: it is the same for subcritical and supercritical case, $u_{t}^{*}(\alpha) \equiv u_{t}^{*}(-\alpha)$.

(2) For large $K$, the probability of extinction in $[0, T]$ is given by

$$
P(\tau \leq T) \approx \exp \left(-\frac{K}{\sigma^{2}} \frac{\alpha}{1-e^{-\alpha T}}\right) .
$$

In particular, for $\alpha=0, P(\tau \leq T) \approx e^{-K / 2 \sigma^{2} T}$.

\section{Proof of Theorem 1.1}

We begin with the following lemma.

Lemma 2.1. For any $n \geq 1, \mathfrak{g}_{n}(-\infty)=\log \mathfrak{f}_{n}(0)$.

Proof. The result follows by induction from the identity $\mathfrak{g}_{n}(\log t) \equiv \log \mathfrak{f}_{n}(t)$ for $t \in\left(0, t_{0}\right)$. Write

$$
\mathfrak{g}(\log (t))=\log \sum_{\ell \geq 0} e^{\ell \log (t)} p_{\ell}=\log \sum_{\ell \geq 0} e^{\log \left(t^{\ell}\right)} p_{\ell}=\log \sum_{\ell \geq 0} t^{\ell} p_{\ell}=\log \mathfrak{f}(t) .
$$

If $\mathfrak{g}_{n-1}(\log t) \equiv \log \mathfrak{f}_{n-1}(t)$, then

$$
\mathfrak{g}_{n}(\log t)=\mathfrak{g}\left(\mathfrak{g}_{n-1}(\log t)\right)=\mathfrak{g}\left(\log \left(\mathfrak{f}_{n-1}(t)\right)\right)=\log \mathfrak{f}\left(\mathfrak{f}_{n-1}(t)\right)=\log \left(\mathfrak{f}_{n}(t)\right)
$$

The proof of Theorem 1.1 is done in a number of steps.

(1) Recall that $\mathfrak{g}(t)$ is convex function with $\mathfrak{g}(0)=0, \mathfrak{g}(-\infty)=\log \left(p_{0}\right)$, and $\mathfrak{g}^{\prime}(t)>$ $0, t>-\infty$, while $\mathfrak{g}^{\prime}(-\infty)=\lim _{t \rightarrow \infty} \mathfrak{g}^{\prime}(t)=0$.

(2)By the local LDP (see, Theorem 4.1), for $u_{0}=1, u_{N}=0$, and other positive $u_{n}$ 's, it holds that

$$
\lim _{\delta \rightarrow 0} \lim _{K \rightarrow \infty} \frac{1}{K} \log P\left(\sum_{n \leq N}\left|x_{n}^{K}-u_{n}\right| \leq \delta\right)=-\sum_{n \leq N} I\left(u_{n}, u_{n-1}\right) .
$$

(3) In order to find $\left(u_{n}^{*}\right)_{n \leq N}$ such that for $u_{0}=1, u_{n}>0, u_{N}=0$,

$$
\sum_{i \leq n} I\left(u_{i}, u_{i-1}\right) \geq \sum_{i \leq n} I\left(u_{i}^{*}, u_{i-1}^{*}\right)
$$

we apply the dynamic programming.

Since $u_{N}=0$,

$$
I\left(u_{N}, u_{N-1}\right)=\sup _{t \in\left(-\infty, t_{0}\right)}\left(-u_{N-1} \mathfrak{g}(t)\right)=-u_{N-1} \mathfrak{g}(-\infty)=: B_{n}\left(u_{N-1}\right)
$$

is the boundary condition for the Bellman equation

$$
B_{n}\left(u_{n-1}\right)=\inf _{u>0}\left[B_{n+1}(u)+I\left(u, u_{n-1}\right)\right], \quad 1 \leq n \leq N-1 .
$$


For $n=N-1$, we have

$$
B_{N-1}\left(u_{N-2}\right)=\inf _{u>0}\left[-u \mathfrak{g}(-\infty)+\sup _{t \in\left(-\infty, t_{0}\right)}\left\{t u-u_{N-2} \mathfrak{g}(t)\right\}\right] .
$$

Equation (2.7) provides the inequality

$$
B_{N-1}\left(u_{N-2}\right) \geq \inf _{u>0}\left[-u \mathfrak{g}(-\infty)+t u-u_{N-2} \mathfrak{g}(t)\right] \quad \forall t \in\left(-\infty, t_{0}\right)
$$

which, with $t=\mathfrak{g}(-\infty)$, is transformed into

$$
B_{N-1}\left(u_{N-2}\right) \geq-u_{N-2} \mathfrak{g}_{2}(-\infty)
$$

We show that the above inequality is equality. For $u, u_{N-2}>0$, "sup $t_{t}$ in (2.7) is attained at the point $t^{*}=t^{*}\left(u, u_{N-2}\right)$, so that, for any $u>0$,

$$
B_{N-1}\left(u_{N-2}\right) \leq u\left[t^{*}\left(u, u_{N-2}\right)-\mathfrak{g}(-\infty)\right]-u_{N-2} \mathfrak{g}\left(t^{*}\left(u, u_{N-2}\right)\right) .
$$

We choose $u=u_{N-1}^{*}$ such that $t^{*}\left(u_{N-1}^{*}, u_{N-2}\right)=\mathfrak{g}(-\infty)$. This is possible since

$$
\begin{gathered}
\mathfrak{g}(-\infty)=\log p_{0}, \quad t^{*}\left(0, u_{N-2}\right)=-\infty, \\
\mathfrak{g}^{\prime}(-\infty)=0, \quad t^{*}\left(\mathfrak{m}, u_{n-2}\right)=0, \quad \mathfrak{g}^{\prime}(0)=\mathfrak{m},
\end{gathered}
$$

so that the existence of $u_{N-1}^{*}$ follows from continuity, in $u$, of $t^{*}\left(u, u_{N-2}\right)$.

The choice of $u_{N-1}^{*}$ gives the inequality

$$
B_{N-1}\left(u_{N-2}\right) \leq-u_{N-2} \mathfrak{g}\left(t^{*}\left(u_{N-1}^{*}, u_{N-2}\right)\right)=\mathfrak{g}(\mathfrak{g}(-\infty))=\mathfrak{g}_{2}(-\infty)
$$

Consequently, the opposite inequality for (2.9) holds true and, therefore,

$$
B_{N-1}\left(u_{N-2}\right)=-u_{N-2} \mathfrak{g}_{2}(-\infty)
$$

It is obvious too that for any $u_{N-2}>0$,

$$
u_{N-1}^{*}=u_{N-2} \mathfrak{g}^{\prime}\left(t^{*}\left(u_{N-1}^{*}, u_{N-2}\right)\right)=u_{N-2} \mathfrak{g}^{\prime}(\mathfrak{g}(-\infty)) .
$$

Further, by induction, we find the following pairs:

$$
\begin{gathered}
u_{N-1}^{*}=\mathfrak{g}^{\prime}(\mathfrak{g}(-\infty)) u_{N-2}^{*}, \\
B_{N-1}\left(u_{N-2}^{*}\right)=-\mathfrak{g}_{2}(-\infty) u_{N-2}^{*}, \\
\ldots \\
u_{N-2}^{*}=\mathfrak{g}^{\prime}\left(\mathfrak{g}_{2}(-\infty)\right) u_{N-3}^{*}, \\
B_{N-2}\left(u_{N-3}^{*}\right)=-\mathfrak{g}_{3}(-\infty) u_{N-3}^{*}, \\
\vdots \\
u_{1}^{*}=\mathfrak{g}^{\prime}\left(\mathfrak{g}_{n-1}(-\infty)\right) u_{0}, \\
B_{1}\left(u_{0}\right)=-\mathfrak{g}_{n}(-\infty) u_{0}\left(u_{0}=1\right) .
\end{gathered}
$$


With the chosen $\left(u_{n}^{*}\right)_{1 \leq n \leq N-1}$, the Bellman equation (2.6) is transformed into the backward recurrent equation

$$
B_{n}\left(u_{n-1}^{*}\right)=B_{n+1}\left(u_{n}^{*}\right)+I\left(u_{n}^{*}, u_{n-1}^{*}\right), \quad 1 \leq n \leq N-1,
$$

with boundary condition $-u_{N-1}^{*} \mathfrak{g}(-\infty)($ see $(2.5))$.

Thus, $B_{1}(1)=\sum_{1 \leq n \leq N} I\left(u_{n}^{*}, u_{n-1}^{*}\right)$.

On the other hand, the Bellman equation also yields

$$
B_{1}(1) \geq \sum_{1 \leq n \leq N-1} I\left(u_{n}, u_{n-1}\right)+B_{N}\left(u_{N-1}\right)=\sum_{1 \leq n \leq N} I\left(u_{n}, u_{n-1}\right)
$$

which proves (2.4).

(4) We recall that $\sum_{n \leq N} I\left(u_{n}^{*}, u_{n-1}^{*}\right)=-\mathfrak{g}_{n}(-\infty)$, that is, by Lemma 2.1 and (1.3),

$$
\sum_{1 \leq n \leq N} I\left(u_{n}^{*}, u_{n-1}^{*}\right)=-\log \mathfrak{f}_{N}(0)=-\frac{1}{K} \log P(\tau \leq N) \quad \forall K>0 .
$$

(5) Thus, (1)-(3) imply the statement (i); formula (1.11) follows from the recurrence $u_{n}^{*}=\mathfrak{g}^{\prime}\left(\mathfrak{g}_{2}(-\infty)\right) u_{n-1}^{*}, \mathfrak{u}_{0}^{*}=1$.

Finally (ii) follows from (4).

\section{Proof of Theorem 1.2}

(i) We apply the LDP Theorem 5.1. By the local LDP, with $u_{0}=1, u_{t}>0$, and $u_{T}=0$, we have

$$
\lim _{\delta \rightarrow 0} \lim _{K \rightarrow \infty} \frac{1}{K} \log P\left(\sup _{t \leq T}\left|x_{t}^{K}-u_{t}\right| \leq \delta\right)=-J_{T}(u)
$$

where

$$
J_{T}(u)= \begin{cases}\frac{1}{2 \sigma^{2}} \int_{0}^{T} \frac{\left(\dot{u}_{t}-u_{t}\right)^{2}}{u_{t}} I_{\left\{u_{t}>0\right\}} d t, & u_{0}=1, d u_{t}=\dot{u}_{t} d t \\ \infty, & \text { otherwise. }\end{cases}
$$

Therefore (i) is reduced to minimization of $J_{T}(u)$ in a class of absolutely continuous test functions $u_{t}$ with $u_{0}=1, u_{t}>0$, and $u_{T}=0$.

Set $w_{t}=\left(\dot{u}_{t}-u_{t}\right) / \sqrt{u_{t}}, t \in[0, T)$ and notice that the minimization of $J_{T}(u$. $)$ is equivalent to the following control problem with the controlled process $u_{t}$, solving a differential equation

$$
\dot{u}_{t}=\alpha u_{t}+\sqrt{u_{t}} w_{t}, \quad t \in[0, T),
$$

subject to $u_{0}=1$. The control action $w_{t}$ belongs to a class of measurable functions with $\int_{0}^{T} w_{t}^{2} d t<\infty$ bringing $u_{t}$ to zero at the time $T$. The control action $w_{t}^{*}$ from this class is optimal if for any $w_{t}$,

$$
\int_{0}^{T}\left(w_{t}^{*}\right)^{2} d t \leq \int_{0}^{T} w_{t}^{2} d t
$$


If $w_{t}^{*}$ exists, then the controlled process $u_{t}^{*}$ related to $w_{t}^{*}$ minimizes $J_{T}(u$. ) in the required class of continuous functions $u$. $=\left(u_{t}\right)_{t \leq T}$.

In order to find $w_{t}^{*}$, it is convenient to deal with (recall $u_{t} \geq 0$ ) $v_{t}=\sqrt{u_{t}}$ since $v_{t}$ solves the linear differential equation $\dot{v}_{t}=(\alpha / 2) v_{t}+(1 / 2) w_{t}, v_{0}=1$. If $w_{t}^{*}$ exists, then $w_{t}^{*}$ brings $v_{t}$ to zero at the time $T$, that is, $0=v_{T}=e^{(\alpha / 2) T}+\int_{0}^{T} e^{(\alpha / 2)(T-t)} w_{t}^{*} d t$ or, equivalently,

$$
-1=\frac{1}{2} \int_{0}^{T} e^{-(\alpha / 2) t} w_{t}^{*} d t
$$

Hence, by the Cauchy-Schwarz inequality $1 \leq(1 / 2) \int_{0}^{T} e^{-t \alpha} d t \int_{0}^{T}\left(w_{t}^{*}\right)^{2} d t$, that is, the following lower bound holds: $\int_{0}^{T}\left(w_{t}^{*}\right)^{2} d t \geq 2 \alpha /\left(1-e^{-\alpha T}\right)$. This lower bound is valid for any $w_{t}$ providing $(3.5)$, so that the condition

$$
\int_{0}^{T}\left(w_{t}^{*}\right)^{2} d t=\frac{2 \alpha}{1-e^{-\alpha T}}
$$

is valid for $w_{t}^{*}=c e^{-t \alpha / 2}$, for any constant $c$, take $w_{t}^{*}=c^{*} e^{-t \alpha / 2}$ with $c^{*}$ solving

$$
-1=\int_{0}^{T} e^{-t \alpha / 2} w_{t}^{*} d t=c^{*} \int_{0}^{T} e^{-t \alpha} d t .
$$

Hence,

$$
\begin{gathered}
c^{*}=\left\{\begin{array}{ll}
-\frac{2 \alpha}{1-e^{-T \alpha}}, & \alpha \neq 0, \\
-\frac{2}{T}, & \alpha=0,
\end{array}, \quad w_{t}^{*}=\left\{\begin{array}{ll}
-\frac{2 \alpha e^{-t(\alpha) /(2)}}{1-e^{-T \alpha}}, & \alpha \neq 0, \\
-\frac{2}{T}, & \alpha=0,
\end{array},\right.\right. \\
\int_{0}^{T}\left(w_{t}^{*}\right)^{2} d t= \begin{cases}\frac{2 \alpha}{1-e^{-\alpha T}}, & \alpha \neq 0, \\
\frac{2}{T}, & \alpha=0 .\end{cases}
\end{gathered}
$$

Finally, we find that

$$
\begin{aligned}
v_{t}^{*} & =e^{t \alpha / 2}-\frac{\alpha}{1-e^{-T \alpha}} \int_{0}^{t} e^{(t-s) \alpha / 2} e^{-s \alpha / 2} d s \\
& =e^{t \alpha / 2}\left[1-\frac{1-e^{-t \alpha}}{1-e^{-T \alpha}}\right]=e^{t \alpha / 2}\left(\frac{e^{-t \alpha}-e^{-T \alpha}}{1-e^{-T \alpha}}\right)
\end{aligned}
$$

and, since $u_{t}^{*}=\left(v_{t}^{*}\right)^{2}$, we obtain (1.19) and the proof of (i) is complete.

(ii) By (i),

$$
J_{T}\left(u^{*}\right)=\frac{1}{\sigma^{2}} \frac{\alpha}{1-e^{-\alpha T}} .
$$

We show that

$$
\lim _{K \rightarrow \infty} \frac{1}{K} \log P(\tau \leq T)=J_{T}\left(u^{*}\right) .
$$


To this end, use the fact that $\{\tau \leq T\}=\left\{(\omega, t): \exists t \leq T, x_{t}^{K}(\omega)=0\right\}$. For notational convenience denote $\mathfrak{A}:=\{\tau \leq T\}$. Set $\mathfrak{A}^{\mathrm{cl}}$ and $\mathfrak{A}^{\text {int }}$ the closure and interior of $\mathfrak{A}$. Then, by the LDP, we have

$$
\begin{gathered}
\varlimsup_{K \rightarrow \infty} \frac{1}{K} \log P\left(\mathfrak{A}^{\mathrm{cl}}\right) \leq-\inf _{\substack{u: \\
u_{s}>0, s<t ; \\
u_{t}=0 \\
t \leq T}} J_{t}(u)=-\inf _{t \leq T} J_{t}\left(u^{*}\right), \\
\varliminf_{K \rightarrow \infty} \frac{1}{K} \log P\left(\mathfrak{A}^{\text {int }}\right) \geq-\inf _{\substack{u_{s}>0, s<t ; \\
u_{t}=0 \\
t \leq T}} J_{t}(u)=-\inf _{t \leq T} J_{t}\left(u^{*}\right) .
\end{gathered}
$$

Since $\underline{\lim }_{K \rightarrow \infty}=\varlimsup_{K \rightarrow \infty}$ implies the existence of $\lim _{K \rightarrow \infty}$, it remains to show that $\inf _{t \leq T} J_{t}\left(u^{*}\right)=J_{T}\left(u^{*}\right)$.

Notice that (3.10) is valid with $T$ replaced by any $t<T$ with $u^{*}$. replaced by the corresponding $u^{*, t}=\left\{u_{0}^{*, t}=1 ; u_{s}^{*, t}>0, s<t ; u_{t}^{s, t}=0\right\}$. In other words, for any $t$,

$$
J_{t}\left(u^{*, t}\right)=\frac{1}{\sigma^{2}} \frac{\alpha}{1-e^{-\alpha t}}
$$

and $J_{t}\left(u^{*, t}\right)$ increases to $J_{T}\left(u^{*}\right)$ with $t \nearrow T$.

\section{LDP in discrete time}

Let $m=\inf \left\{n \leq N: u_{n}=0\right\}$ and $m=\infty$ if all $\left(u_{n}\right)_{n \leq N}$ are positive,

$$
I(y, x)=\sup _{t \in\left(-\infty, t_{0}\right)}[t y-x \mathfrak{g}(t)] .
$$

Theorem 4.1. Assume (1.8). For any $N \geq 1$, the family $\left\{\left(x_{n}^{K}\right)_{n \leq N}\right\}_{K \rightarrow \infty}$ obeys the LDP in $\mathbb{R}_{+}^{N}$, supplied with the Euclidian metric $\varrho_{N}$, with the speed $1 / K$ and the rate function

$$
J_{N}(u .)= \begin{cases}\sum_{n=1}^{m-1} I\left(u_{n}, u_{n-1}\right)-u_{m-1} \log \left(p_{0}\right), & u_{0}=1, u_{n}=0, n>m, \\ \sum_{n=1}^{N} I\left(u_{n}, u_{n-1}\right), & u_{0}=1, u_{n}>0, n \leq N, \\ \infty, & \exists n: u_{n}=0, u_{n+1}>0, \text { or } u_{0} \neq 1 .\end{cases}
$$

Remark 4.2. LDP for branching processes has been considered in the literature, see, for example, $[1,4,18]$. However, it was concerned with the sequence $X_{n} / X_{n-1}$, as $n \rightarrow \infty$, whereas here we consider the LDP for $X_{n} / X_{0}$ processes indexed by the large initial value.

Remark 4.3. The nonnegativity of $x_{n}^{K}$ provides some difficulty for verification of LDP at the "point of extinction" where the test function becomes zero. For set $\mathbb{S}$ of test functions that keep away from zero, the statement of the theorem is implied by a result in Klebaner and Zeitouni [14] and other known results that can be adapted to our setting (see, e.g., 
Kifer, [8], Puhalskii, [19], Klebaner and Liptser, [11], etc.). But $\{\tau \leq N\} \notin \mathbb{S}$, and for the sake of completeness and accuracy we give the complete proof below, with a new proof of the lower bound in the local LDP.

4.1. Proof of Theorem 4.1. We follow the standard (necessary and sufficient) conditions for proving the LDP by showing the exponential tightness

$$
\lim _{C \rightarrow \infty} \varlimsup_{K \rightarrow \infty} \frac{1}{K} \log P\left(\Omega \backslash \mathscr{K}_{C}\right)=-\infty,
$$

with compacts $\mathscr{K}_{C}=\left\{\max _{1 \leq n \leq N} x_{N} \leq C\right\}, C \succ \infty$, and the local LDP

$$
\lim _{\delta \rightarrow 0} \lim _{K \rightarrow \infty} \frac{1}{K} \log P\left(\varrho_{N}\left(x_{.}^{K}, u .\right) \leq \delta\right)=-J_{N}(u .) .
$$

Notice that (1.8) implies the existence of a stochastic exponential, with $t_{n} \leq K t_{0}$,

$$
\mathscr{E}_{\left(t_{1}, \ldots, t_{N}\right)}\left(x_{1}^{K}, \ldots, x_{N-1}^{K}\right)=\prod_{n=1}^{N} E\left(e^{t_{n} x_{n}^{K}} \mid \mathscr{F}_{n-1}\right),
$$

where $\left(\mathscr{F}_{n}\right)_{n \geq 0}$ is the filtration, with $\mathscr{F}_{0}=\{\varnothing, \Omega\}$, generated by $\left(x_{n}^{K}\right)_{n \geq 1}$.

Set

$$
\mathfrak{z}_{n}=e^{\sum_{i \leq n} t_{e} x_{i}^{K}-\log \mathscr{E}_{\left(t_{1}, \ldots, t_{n}\right)}^{K}\left(x_{1}^{K}, \ldots, x_{n-1}^{K}\right)} .
$$

The random process $\left(\mathfrak{z}_{n}, \mathscr{F}_{n}\right)_{n \leq N}$ is the (positive) martingale,

$$
E_{\mathfrak{z}_{N}}=1 .
$$

4.1.1. Exponential tightness. Since $\max _{1 \leq n \leq N} x_{i}^{K} \leq \sum_{1 \leq n \leq N} x_{n}^{K}$, it is enough to show

$$
\lim _{C \rightarrow \infty} \varlimsup_{K \rightarrow \infty} \frac{1}{K} \log P\left(\sum_{1 \leq i \leq N} x_{i}^{K} \geq C\right)=-\infty .
$$

Set $t^{*}=\operatorname{argmax}_{t \in\left(-\infty, t_{0}\right)}[t-\mathfrak{g}(t)]$. Since $\mathfrak{g}(0)=0$, we have $t^{*} \in\left(0, t_{0}\right)$ and $\mathfrak{g}\left(t^{*}\right)<t^{*}$. We choose $t_{n} \equiv t^{*} K\left(<K t_{0}\right)$ and introduce $\mathfrak{A}=\left\{\sum_{1 \leq i \leq n} x_{i}^{K} \geq C\right\}$. With chosen $t_{n}$, we have $E \mathfrak{z}_{N}=1$ and, therefore, $E I_{\mathfrak{A} \mathfrak{z}_{N}} \leq 1$. Taking into account this inequality and (4.6), write

$$
\begin{aligned}
1 & \geq E I_{\mathfrak{A}} e^{\sum_{\{1 \leq n \leq N\}} t^{*} x_{n}^{K}-\log \mathscr{E}_{\left(t^{*}, \ldots, t^{*}\right)}^{K}\left(x_{1}^{K}, \ldots, x_{N-1}^{K}\right)} \\
& =E I_{\mathfrak{A}} e^{K t^{*} \sum_{\{1 \leq n \leq N\}} x_{n}^{K}-K \mathfrak{g}\left(t^{*}\right) \sum_{\{1 \leq n \leq N\}} x_{n-1}^{K}} \\
& \geq E I_{\mathfrak{A}} e^{K \sum_{\{1 \leq n \leq N\}}\left[t^{*}-\mathfrak{g}\left(t^{*}\right)\right] x_{n}^{K}-K\left|\mathfrak{g}\left(t^{*}\right)\right|} \\
& \geq E I_{\mathfrak{A}} e^{K C\left[t^{*}-\mathfrak{g}\left(t^{*}\right)\right]}=e^{K C\left[t^{*}-\mathfrak{g}\left(t^{*}\right)\right]-K\left|\mathfrak{g}\left(t^{*}\right)\right|} P(\mathfrak{A}) .
\end{aligned}
$$

Therefore, $1 / K \log P(\mathfrak{A}) \leq-\underbrace{\left[t^{*}-\mathfrak{g}\left(t^{*}\right)\right]}_{>0} C+\left|\mathfrak{g}\left(t^{*}\right)\right| \underset{C \rightarrow \infty}{\longrightarrow}-\infty$. 
4.1.2. Local LDP: upper bound. We may restrict ourselves by the test function $u$. $=$ $\{\underbrace{u_{1}, \ldots, u_{N-1}}_{>0}, \underbrace{u_{N}}_{=0}\}$ and show that

$$
\varlimsup_{\delta \rightarrow 0} \varlimsup_{K \rightarrow \infty} \frac{1}{K} \log P\left(\varrho_{N}\left(x_{.}^{K}, u .\right) \leq \delta\right) \leq-J_{N}(u .) .
$$

For the test function with all positive $u_{n}$ 's and $u_{0}=1$, the proof of (4.10) is similar. For test function with $u_{n}=0, u_{n+1}>0$, or $u_{0} \neq 1,(4.10)$ is obvious. For other test functions, the verification of (4.10) is reduced to the above-mentioned ones.

Let now $\mathfrak{A}=\left\{\rho_{N}\left(x^{K}, u.\right) \leq \delta\right\}$. By $(4.7)$, we have

$$
1 \geq E I_{\mathfrak{A} \mathfrak{z} N}=E I_{\mathfrak{A}} e^{\sum_{\{1 \leq n \leq N\}}\left[t_{n} x_{i}^{K}-K x_{n-1}^{K} \mathfrak{g}\left(t_{n} / K\right)\right]} .
$$

Set $t_{n}^{*}=\operatorname{argmax}_{t \in\left(-\infty, t_{0}\right)}\left[t u_{n}-u_{n-1} \mathfrak{g}(t)\right], n \leq N-1$, and $t_{N}^{*}=-l(l>0)$, and take $t_{n}=$ $K t_{n}^{*}$, then we derive from (4.11)

$$
\begin{aligned}
1 & \geq E I_{\mathfrak{A}} e^{K \sum_{\{1 \leq n \leq N\}}\left[t_{n}^{*} u_{n}-u_{n-1} \mathfrak{g}\left(t_{n}^{*}\right)\right]-K \sum_{1 \leq n \leq N-1}\left(t_{n}^{*}+\left|\mathfrak{g}\left(t_{n}^{*}\right)\right|\right) \delta} \\
& =E I_{\mathfrak{A}} e^{K\left[\sum_{\{1 \leq n \leq N-1\}} I\left(u_{n}, u_{n-1}\right)-u_{N-1} \mathfrak{g}(-l)\right]-K \sum_{1 \leq n \leq N-1}\left(\left|t_{n}^{*}\right|+\left|\mathfrak{g}\left(t_{n}^{*}\right)\right|\right) \delta} \\
& =E I_{\mathfrak{A}} e^{K\left[J_{N-1}\left(u_{.}\right)-u_{N-1} \mathfrak{g}(-l)\right]-K \sum_{1 \leq n \leq N-1}\left(\left|t_{n}^{*}\right|+\left|\mathfrak{g}\left(t_{n}^{*}\right)\right|\right) \delta} .
\end{aligned}
$$

Hence, taking into account that $\lim _{l \rightarrow \infty} \mathfrak{g}(-l)=\log \left(p_{0}\right)$, we obtain

$$
\begin{aligned}
\frac{1}{K} \log P(\mathfrak{A}) & \leq-\left[J_{N-1}(u .)+u_{N-1} \mathfrak{g}(-l)\right]+\sum_{1 \leq i \leq N-1}\left(\left|t_{i}^{*}\right|+\left|\mathfrak{g}\left(t_{i}^{*}\right)\right|\right) \delta \\
& \underset{\delta \rightarrow 0}{\longrightarrow}-\left[J_{N-1}(u .)+u_{N-1} \mathfrak{g}(-l)\right] \underset{l \rightarrow \infty}{\longrightarrow}-J_{N}(u .) .
\end{aligned}
$$

4.2. Local LDP: lower bound. Obviously for $u$. with $J_{N}(u$. $)=\infty$, it is nothing to verify. Further as in the upper bound verification, we may restrict ourselves by the test function $u .=\{\underbrace{u_{1}, \ldots, u_{N-1}}_{>0}, \underbrace{u_{N}}_{=0}\}$ with $P\left(\xi_{1}^{1}=0\right)=p_{0}>0$ and show that

$$
\varliminf_{\delta \rightarrow 0} \frac{\lim }{K \rightarrow \infty} \frac{1}{K} \log P\left(\varrho_{N}\left(x_{.}^{K}, u .\right) \leq \delta\right) \geq-J_{N}(u .) .
$$

Write

$$
\begin{aligned}
& \left\{\varrho_{N}\left(x_{.}^{K}, u .\right) \leq \delta\right\} \\
& \quad=\left\{\varrho_{N-1}\left(x_{.}^{K}, u .\right)+x_{N}^{K} \leq \delta\right\} \supseteq\left\{\varrho_{N-1}\left(x_{.}^{K}, u .\right) \leq 0.5 \delta, x_{N}^{K} \leq 0.5 \delta\right\} \\
& \quad \supseteq\left\{\varrho_{N-1}\left(x_{.}^{K}, u .\right) \leq 0.5 \delta, x_{N}^{K}=0\right\} \supseteq\left\{\varrho_{N-1}\left(x_{.}^{K}, u .\right) \leq 0.5 \delta, \frac{1}{K} \sum_{j=1}^{K x_{N-1}^{K}} \xi_{N}^{j}=0\right\} \\
& \quad \supseteq\left\{\varrho_{N-1}\left(x_{.}^{K}, u .\right) \leq 0.5 \delta, \frac{1}{K} \sum_{j=1}^{K\left(u_{N-1}+\delta\right)} \xi_{N}^{j}=0\right\}=\left\{\varrho_{N-1}\left(x_{.}^{K}, u .\right) \leq 0.5 \delta, \sum_{j=1}^{K\left(u_{N-1}+\delta\right)} \xi_{N}^{j}=0\right\} .
\end{aligned}
$$


The sets $\mathfrak{A}_{1}=\left\{\varrho_{N-1}\left(x^{K}, u.\right) \leq 0.5 \delta\right\}$ and $\mathfrak{A}_{2}=\left\{\sum_{j=1}^{K\left(u_{N-1}+\delta\right)} \xi_{N}^{j}=0\right\}$ are independent, so that

$$
P\left(\varrho_{N}\left(x^{K}, u .\right) \leq \delta\right) \geq P\left(\varrho_{N-1}\left(x_{.}^{k}, u .\right) \leq 0.5 \delta\right) P^{K\left(u_{N-1}+\delta\right)}\left(\xi_{1}^{1}=0\right) .
$$

Consequently,

$$
\begin{aligned}
& \frac{\lim _{\delta \rightarrow 0}}{\lim _{K \rightarrow \infty}} \frac{1}{K} \log P\left(\varrho_{N}\left(x_{.}^{K}, u .\right) \leq \delta\right) \\
& \quad \geq \frac{\lim }{\delta \rightarrow 0} \frac{\lim }{K \rightarrow \infty} \frac{1}{K} \log P\left(\varrho_{N-1}\left(x_{.}^{K}, u .\right) \leq 0.5 \delta\right)+u_{N-1} \log P\left(\xi_{1}^{1}=0\right) .
\end{aligned}
$$

If

$$
\varliminf_{\delta \rightarrow 0} \frac{\lim _{K \rightarrow \infty}}{K} \frac{1}{K} \log P\left(\varrho_{N-1}\left(x^{K}, u .\right) \leq \delta\right) \geq-J_{N-1}(u .),
$$

provided that $u_{n}>0, n \leq N-1$, the required lower bound holds true.

Thus, it is left to verify the validity of (4.18).

Set $\Lambda_{N-1}\left(x^{K}\right)=\mathfrak{z}_{N-1}$, that is,

$$
\Lambda_{N-1}\left(x^{K}\right)=e^{\sum_{n=1}^{N-1} K\left[t_{n}^{*} x_{n}^{K}-x_{n-1}^{K} \mathfrak{g}\left(t_{n}^{*}\right)\right]}, \quad E \Lambda_{N-1}\left(x_{.}^{K}\right)=1 .
$$

We introduce the probability measure $Q_{N-1}^{K}$ with $d Q_{N-1}^{K}=\Lambda_{N-1}\left(x^{K}\right) d P$. Since $\Lambda_{n-1}\left(x^{K}\right)>0, P$-a.s., we also have $d P=\Lambda_{n-1}^{-1}\left(x^{K}\right) d Q_{n-1}^{K}$.

In particular, for $\mathfrak{A}=\left\{\varrho_{N-1}\left(x_{.}^{K}, u_{.}\right) \leq \delta\right\}$,

$$
P(\mathfrak{A})=\int_{\mathfrak{A}} \Lambda_{N-1}^{-1}\left(x^{K}\right) d Q_{N-1}^{K} .
$$

So, the following lower bound, on the set $\mathfrak{A}$, is valid:

$$
\begin{aligned}
\Lambda_{N-1}^{-1}\left(x_{.}^{K}\right) & \geq e^{-K J_{N-1}(u .)-K \delta \max _{n \leq N-1}\left(\left|t_{n}^{*}\right|+\left|\mathfrak{g}\left(t_{n}^{*}\right)\right|\right)} \\
& \geq e^{-K J_{N-1}(u .)-K \delta \max _{n \leq N-1}\left(\left|t^{a} s t_{n}\right|+\left|\mathfrak{g}\left(t_{n}^{*}\right)\right|\right)}
\end{aligned}
$$

or, equivalently,

$$
\frac{1}{K} \log P(\mathfrak{A}) \geq-J_{N-1}(u .)-\delta \max _{n \leq N-1}\left(\left|t_{n}^{*}\right|+\left|\mathfrak{g}\left(t_{n}^{*}\right)\right|\right)+\frac{1}{K} \log Q_{N-1}^{K}(\mathfrak{A}) .
$$

The latter inequality implies (4.18) if

$$
\lim _{K \rightarrow \infty} \frac{1}{K} \log Q_{N-1}^{K}(\mathfrak{A})=0 .
$$

A simple condition, providing (4.23), is $\lim _{K \rightarrow \infty} Q_{N-1}^{K}(\mathfrak{A})=1$ or, equivalently,

$$
\lim _{K \rightarrow \infty} Q_{N-1}^{K}\left(\varrho_{N-1}\left(x_{.}^{K}, u .\right)>\delta\right)=0 .
$$


We verify (4.24) by showing ( $E_{N-1}^{K}$ denotes the expectation with respect to $Q_{N-1}^{K}$ )

$$
E_{N-1}^{K} \varrho_{N-1}^{2}\left(x^{K} \cdot, u .\right)=\frac{u_{N-1}}{K} \sum_{n=1}^{N-1} \frac{u_{n-1}}{u_{n}^{2}} \mathfrak{g}^{\prime \prime}\left(t_{n}^{*}\right) .
$$

Notice that the positiveness of $\left(u_{n}\right)_{n \leq N-1}$ provides a boundedness for the right-hand side of (4.25) and, in turn by Chebyshev's inequality, the validity of (4.24).

In order to establish (4.25), we apply the identity relative to $t_{n}^{*}$ :

$$
1=E\left(\frac{\Lambda_{n}\left(x^{K}\right)}{\Lambda_{n-1}\left(x^{K}\right)} \mid \mathscr{F}_{n-1}\right)=E e^{K\left[t_{n}^{*} x_{n}^{K}-x_{n-1}^{K} \mathfrak{g}\left(t_{n}^{*}\right)\right]} .
$$

Differentiating twice $(4.26)$ in $t_{n}^{*}$, we find that

$$
\begin{gathered}
0=E\left(\left[x_{n}^{K}-x_{n-1}^{K} \mathfrak{g}^{\prime}\left(t_{n}^{*}\right)\right] \frac{\Lambda_{i}\left(x_{\cdot}^{K}\right)}{\Lambda_{n-1}\left(x_{*}^{K}\right)} \mid \mathscr{F}_{n-1}\right), \\
0=E\left(\left\{K\left[x_{n}^{K}-x_{n-1}^{K} \mathfrak{g}^{\prime}\left(t_{n}^{*}\right)\right]^{2}-x_{n-1}^{K} \mathfrak{g}^{\prime \prime}\left(t_{n}^{*}\right)\right\} \frac{\Lambda_{n}\left(x_{.}^{K}\right)}{\Lambda_{n-1}\left(x_{.}^{K}\right)} \mid \mathscr{F}_{n-1}\right) .
\end{gathered}
$$

By the Bayes formula, for example, $[10,16]$, for any integrable random variable $\alpha$,

$$
E_{N-1}^{K}\left(\alpha \mid \mathscr{F}_{n-1}\right)=E\left(\alpha \frac{\Lambda_{n}\left(x^{K}\right)}{\Lambda_{n-1}\left(x^{K}\right)} \mid \mathscr{F}_{n-1}\right) .
$$

By taking $\alpha=x_{n}^{K}$ and $\alpha=\left[x_{n}^{K}-x_{n-1}^{K} \mathfrak{g}^{\prime}\left(t_{i}^{*}\right)\right]^{2}$, we derive with the help of (4.27) that

$$
\begin{gathered}
E_{N-1}^{K}\left(x_{n}^{K} \mid \mathscr{F}_{n-1}\right)=x_{n-1}^{K} \mathfrak{g}^{\prime}\left(t_{n}^{*}\right), \\
E_{N-1}^{K}\left(\left[x_{n}^{K}-x_{n-1}^{K} \mathfrak{g}^{\prime}\left(t_{n}^{*}\right)\right]^{2} \mid \mathscr{F}_{n-1}\right)=x_{n-1}^{K} \frac{\mathfrak{g}^{\prime \prime}\left(t_{n}^{*}\right)}{K} .
\end{gathered}
$$

Since $u_{n}, u_{n-1}$ are positive, we have $\mathfrak{g}^{\prime}\left(t_{n}^{*}\right)=u_{n} / u_{n-1}$. Hence, and by (4.29), we obtain that $E_{N-1}^{K} x_{n}^{K}=\left(u_{n} / u_{i-1}\right) E_{N-1}^{K} x_{n-1}^{K}$. Consequently, iterating the above recursion and taking into account $u_{0}=1$, we find that

$$
E_{N-1}^{K} x_{n}^{K}=u_{n}
$$

Further, with the help of (4.30) we find a recursion

$$
E_{N-1}^{K}\left(x_{n}^{K}\right)^{2}=\left(\frac{u_{n}}{u_{n-1}}\right)^{2} E_{N-1}^{K}\left(x_{n-1}^{K}\right)^{2}+u_{n-1} \frac{\mathfrak{g}^{\prime \prime}\left(t_{n}^{*}\right)}{K} .
$$

By using $E_{N-1}^{K}\left(x_{n}^{K}-u_{n}\right)^{2}=E_{N-1}^{K}\left(x_{n}^{K}\right)^{2}-u_{n}^{2}$ and $u_{n}^{2}=\left(u_{n} / u_{n-1}\right)^{2} u_{n-1}^{2}$, we establish a recursion for $\triangle_{n}=E_{N-1}^{K}\left(x_{n}^{K}-u_{n}\right)^{2}$ :

$$
\triangle_{n}=\left(\frac{u_{n}}{u_{n-1}}\right)^{2} \triangle_{n-1}+u_{n-1} \frac{\mathfrak{g}^{\prime \prime}\left(t_{n}^{*}\right)}{K}
$$


supplied by $\triangle_{0}=0$. Then, $\triangle_{0} / u_{0}^{2}=0$ and

$$
\frac{\triangle_{n}}{u_{n}^{2}}=\frac{\triangle_{n-1}}{u_{n-1}^{2}}+\frac{u_{n-1}}{u_{n}^{2}} \frac{\mathfrak{g}^{\prime \prime}\left(t_{n}^{*}\right)}{K}, \quad \triangle_{N-1}=\frac{u_{N-1}}{K} \sum_{n=1}^{N-1} \frac{u_{n-1}}{u_{n}^{2}} \mathfrak{g}^{\prime \prime}\left(t_{n}^{*}\right) .
$$

It is left to recall that $\triangle_{N-1}=E_{N-1}^{K} \varrho_{N-1}^{2}\left(x^{K} \cdot u.\right)$.

\section{LDP in continuous time}

We introduce the filtration $\left(\mathscr{F}_{t}^{B}\right)_{t \geq 0}$ generated by Brownian motion $B_{t}$ with the general conditions. All random processes considered in this section are adapted to this filtration. Henceforth, by agreement,

$$
\frac{0}{0}=0
$$

Theorem 5.1. For any $T>0$, the family $\left\{\left(x_{t}^{K}\right)_{t \leq T}\right\}_{K \rightarrow \infty}$ obeys the LDP in $\mathbb{C}_{[0, T]}\left(\mathbb{R}_{+}\right)$, supplied with the uniform metric $\varrho_{T}$, with the speed $1 / K$ and the rate function

$$
J_{T}(u .)= \begin{cases}\frac{1}{2 \sigma^{2}} \int_{0}^{T} \frac{\left(\dot{u}_{t}-\alpha u_{t}\right)^{2}}{u_{t}} d t, & u_{0}=1, d u_{t}=\dot{u}_{t} d t \\ \infty, & \text { otherwise. }\end{cases}
$$

Remark 5.2. Since $u_{t} \geq 0$, Freidlin-Wentzell's rate function [6] $\left(1 / 2 \sigma^{2}\right) \int_{0}^{T}\left(\left(\dot{u}_{t}-\alpha u_{t}\right)^{2} /\right.$ $\left.u_{t}\right) d t$ is not compatible with $u_{t}=0$. Our branching diffusion model is a very particular case of a model studied by Puhalskii in [20]. To apply the LDP analysis from [20] to the family $\left\{\left(x_{t}^{K}\right)_{t \leq T}\right\}_{K \rightarrow \infty}$, one has to "disentangle" many details of the proof to make it compatible with our case. Finally, in Donati-Martin et al., [5], the LDP analysis deals with a rate function of the following type $\int_{0}^{T}\left(\left(\dot{u}_{t}-\rho\right)^{2} / u_{t}\right) d t$ for $u_{t} \geq 0$ related to a family of diffusion type processes without extinction. A reader interested in the details of the direct proof can find them below.

Proof. It suffices to verify

(i) C-exponential tightness (see [15]),

$$
\begin{gathered}
\lim _{C \rightarrow \infty} \varlimsup_{K \rightarrow \infty} \frac{1}{K} \log P\left(\sup _{t \leq T} x_{t}^{K} \geq C\right)=-\infty, \\
\lim _{\Delta \rightarrow 0} \varlimsup_{K \rightarrow \infty} \sup _{\gamma \leq T} \frac{1}{K} \log P\left(\sup _{t \leq \Delta}\left|x_{\gamma+t}^{K}-x_{\gamma}^{K}\right| \geq \eta\right)=-\infty \quad \forall \eta>0,
\end{gathered}
$$

where $\gamma$ is a stopping time relative to $\left(\mathscr{F}_{t}^{B}\right)_{t \geq 0}$,

(ii) the Local LDP,

$$
\lim _{\delta \rightarrow 0} \lim _{K \rightarrow \infty} \frac{1}{K} \log P\left(\sup _{t \leq T}\left|x_{t}^{K}-u_{t}\right| \leq \delta\right)=-J_{T}(u .) .
$$


(i) Verification. The Itô equation (1.16) is equivalent to the integral equation $x_{t}^{K}=$ $e^{\alpha t}\left(1+(1 / \sqrt{K}) \int_{0}^{t} e^{-\alpha s} \sqrt{x_{s}^{K}} d B_{s}\right)$. Hence,

$$
\sup _{t \leq T} x_{t}^{K} \leq 2 e^{|\alpha| T}\left(1 \vee \frac{\sigma}{\sqrt{K}} \sup _{t \leq T} \int_{0}^{t} e^{-\alpha s} \sqrt{x_{s}^{K}} d B_{s}\right),
$$

so that (5.3) holds true provided that

$$
\lim _{C \rightarrow \infty} \varlimsup_{K \rightarrow \infty} \frac{1}{K} \log P\left(\sup _{t \leq T} \int_{0}^{t} e^{-\alpha s} \sqrt{x_{s}^{K}} d B_{s} \geq \sqrt{K} C\right)=-\infty .
$$

In order to verify (5.7), let us introduce a continuous martingale and its variation process

$$
M_{t}=\frac{\sigma}{\sqrt{K}} \int_{0}^{t} e^{-\alpha s} \sqrt{x_{s}^{K}} d B_{s}, \quad\langle M\rangle_{t}=\frac{\sigma^{2}}{K} \int_{0}^{t} e^{-2 \alpha s} x_{s}^{K} d s,
$$

respectively, and the stopping time $\tau_{C}=\inf \left\{t \leq T: M_{t} \geq C\right\}$, where $\inf \{\varnothing\}=\infty$ which enables us to claim that (5.7) is valid if

$$
\lim _{C \rightarrow \infty} \varlimsup_{K \rightarrow \infty} \frac{1}{K} \log P\left(\tau_{C} \leq T\right)=-\infty .
$$

We proceed with the verification of (5.9). With $\lambda>0$, set

$$
\mathfrak{z}_{t}=e^{\lambda M_{t}-(1 / 2)\langle M\rangle_{t}} .
$$

It is well known that the process $\left(\mathfrak{z}_{t}, \mathscr{F}_{t}^{B}\right)_{t \geq 0}$ is the positive local martingale and so the supermartingale too with $E_{\mathfrak{z} \theta} \leq 1$ for any stopping time $\theta$ relative to $\left(\mathscr{F}_{t}^{B}\right)$. By choosing $\theta=\tau_{C}$, we find that $1 \geq E I_{\{\theta \leq T\}} \mathfrak{z} \theta$. Then, due to a lower bound on the set $\{\theta \leq T\}: \log \mathfrak{z} \theta \geq$ $\lambda C-\left(\sigma^{2} \lambda^{2} / 2 K\right) \int_{0}^{\theta} e^{-2 \alpha s} x_{s}^{K} d s$ and (5.6), there exists positive $l$ such that

$$
\log \mathfrak{z}_{\theta} \geq \lambda C-\frac{\sigma^{2} \lambda^{2}}{2 K} \int_{0}^{\theta} e^{-2 \alpha s} \geq \lambda C-\frac{l \lambda^{2}}{2 K}(1+C) .
$$

Further, a choice of $\lambda=K C /(1+C) l$ implies $\mathfrak{z} \theta \geq e^{K C^{2} /(1+C) l}$. Consequently,

$$
\frac{1}{K} \log P\left(\tau_{C} \leq T\right) \leq-\frac{C^{2}}{(1+C) l} \underset{C \rightarrow \infty}{\longrightarrow}-\infty
$$

By (5.3), the proof of (5.4) is reduced to the verification of two conditions: for any $\eta$, $C>0$,

$$
\begin{gathered}
\lim _{\Delta \rightarrow 0} \varlimsup_{K \rightarrow \infty} \sup _{\gamma \leq T} \frac{1}{K} \log P\left(\sup _{t \leq \Delta} \int_{\gamma}^{\gamma+t} x_{s}^{K} d s \geq \eta, \sup _{s \leq T} x_{s}^{K} \leq C\right)=-\infty, \\
\lim _{\Delta \rightarrow 0} \varlimsup_{K \rightarrow \infty} \sup _{\gamma \leq T} \frac{1}{K} \log P\left(\frac{\sigma}{\sqrt{K}} \sup _{t \leq \Delta}\left|\int_{\gamma}^{\gamma+t} \sqrt{x_{s}^{K}} d B_{s}\right| \geq \eta, \sup _{s \leq T} x_{s}^{K} \leq C\right)=-\infty .
\end{gathered}
$$


The first is obvious while the second is equivalent to

$$
\lim _{\Delta \rightarrow 0} \varlimsup_{K \rightarrow \infty} \sup _{\gamma \leq T} \frac{1}{K} \log P\left(\sup _{t \leq \Delta} I_{T, C}\left|M_{\gamma+t}^{K}-M_{\gamma}^{K}\right| \geq \eta\right)=-\infty \text {, }
$$

where $I_{t, C}=I_{\left\{\sup _{s \leq t} x_{s}^{K} \leq C\right\}}, t \leq T$.

Set $N_{t}^{K}=M_{\gamma+t}^{K}-M_{\gamma}^{K}$ and notice that $\left(N_{t}^{K}, \mathscr{F}_{\gamma+t}^{B}\right)_{t \geq 0}$ is a local martingale with the variation process $\left\langle N^{K}\right\rangle_{t}=\left(\sigma^{2} / K\right) \int_{\gamma}^{\gamma+t} x_{s}^{K} d s$.

Further, the use of $I_{T, C} N_{t}^{K}=I_{T, C} \int_{0}^{t} I_{s, C} d N_{s}^{K}$ simplifies (5.14) to

$$
\lim _{\Delta \rightarrow 0} \varlimsup_{K \rightarrow \infty} \sup _{\gamma \leq T} \frac{1}{K} \log P\left(\sup _{t \leq \Delta}\left|\int_{0}^{t} I_{s, C} d N_{s}^{K}\right| \geq \eta\right)=-\infty .
$$

The local martingale $N_{t}^{K, C}:=\int_{0}^{t} I_{s, C} d N_{s}^{K}$ possesses the variation process

$$
\left\langle N^{K, C}\right\rangle_{t}=\int_{0}^{t} I_{s, C} d\left\langle N^{K}\right\rangle_{s}=\frac{\sigma^{2}}{K} \int_{0}^{t} I_{s, C} x_{s}^{K} d s,
$$

that is, $\mathrm{d}\left\langle N^{K, C}\right\rangle_{t} \leq\left(\sigma^{2} C / K\right) d t$.

Now, we are able to verify (5.15) with the help of stochastic exponential technique. Let

$$
\mathfrak{z}_{t}(\lambda)=e^{\lambda N_{t}^{K, C}-\left(\lambda^{2} / 2\right)\left\langle N^{K, C}\right\rangle_{t}}, \quad \lambda \in \mathbb{R} .
$$

Since $z_{t}(\lambda)$ is a continuous local martingale and supermartingale too, for any stopping time $\theta, E_{\mathfrak{z} \theta}(\lambda) \leq 1$. Let $\theta=\inf \left\{t \leq \Delta: N_{t}^{K, C} \geq \eta\right\}$. Taking into account that $\{\theta \leq \Delta\}=$ $\left\{N_{\theta}^{K, C} \geq \eta\right\}$, write $1 \geq E I_{\{\theta \leq \Delta\}} \mathfrak{z} \theta_{\theta}(\lambda)$. The value $\mathfrak{z}_{\theta}(\lambda)$ is evaluated below on the set $\{\theta \leq \Delta\}$ as follows: with $\lambda>0$ and $\left\langle N^{K, C}\right\rangle_{\theta} \leq\left(\sigma^{2} C / K\right) \theta \leq\left(\sigma^{2} C / K\right) \Delta$,

$$
\mathfrak{z}_{\theta}(\lambda) \geq e^{\lambda \eta-\left(\lambda^{2} \sigma^{2} C / 2 K\right) \Delta} .
$$

Therefore, $\log P(\theta \leq \Delta) \leq-\left[\lambda \eta-\left(\lambda^{2} \sigma^{2} C / 2 K\right) \Delta\right]$ and the choice of $\lambda=K \eta / \sigma^{2} C \Delta$ provides

$$
\frac{1}{K} \log P(\theta \leq \Delta) \leq-\frac{\eta^{2}}{2 \sigma^{2} C \Delta} \underset{\Delta \rightarrow 0}{\longrightarrow}-\infty
$$

It is clear that the same result remains valid for $\theta=\inf \left\{t:-N_{t}^{K, C} \geq \eta\right\}$. Combining both, we obtain (5.15).

(ii) Verification. The upper bound. For $u_{0} \neq 1$ or $d u_{t} \nless d t$, the proof is obvious. For $u_{0}=$ 1 and $d u_{t}=\dot{u}_{t} d t$, the stochastic exponential technique is applicable. With an absolutely continuous deterministic function $\lambda(t)$, let us introduce a continuous martingale $M_{t}$ and its predictable variation process $\langle M\rangle_{t}$ :

$$
M_{t}=\frac{\sigma}{\sqrt{K}} \int_{0}^{t} \lambda(s) \sqrt{x_{s}^{K}} d B_{s}, \quad\langle M\rangle_{t}=\frac{\sigma^{2}}{K} \int_{0}^{t} \lambda^{2}(s) x_{s}^{K} d s
$$


It is well known that the stochastic exponential $\mathfrak{z}_{t}=e^{M_{t}-0.5\langle M\rangle_{t}}$ is a local martingale and a supermartingale too with $E \mathfrak{z}_{T} \leq 1$. The use of this property implies

$$
1 \geq E I_{\left\{\sup _{t \leq T}\left|x_{t}^{K}-u_{t}\right| \leq \delta\right\} \mathfrak{z} T}
$$

The next helpful step of the proof gives a deterministic lower bound for $\mathfrak{z}_{T}$ on the set $\left\{\sup _{t \leq T}\left|x_{t}^{K}-u_{t}\right| \leq \delta\right\}=: \mathfrak{A}$. By (1.13), $M_{t}=\int_{0}^{t} \lambda(s)\left(d x_{s}^{K}-\alpha x_{s}^{K} d s\right)$, so that

$$
\begin{aligned}
\log \mathfrak{z}_{T}= & \int_{0}^{T} \lambda(s)\left(d x_{s}^{K}-\alpha x_{s}^{K} d s\right)-\frac{\sigma^{2}}{2 K} \int_{0}^{t} \lambda^{2}(s) x_{s}^{K} d s \\
= & \int_{0}^{T}\left[\lambda(s)\left(\dot{u}_{s}-\alpha u_{s} d s\right)-\frac{\sigma^{2}}{2 K} \lambda^{2}(s) u_{s}\right] d s \\
& +\int_{0}^{T} \lambda(s) d\left(x_{s}^{K}-u_{s}\right)=\left[\lambda_{T}\left(x_{T}^{K}-u_{T}\right)-\int_{0}^{T}\left(x_{s}^{K}-u_{s}\right) \dot{\lambda}_{t} d s\right] \\
& -\int_{0}^{T}\left[\lambda(s) \alpha\left\{x_{s}^{K}-u_{s}\right\}+\frac{\sigma^{2}}{2 K} \lambda^{2}(s)\left\{x_{s}^{K}-u_{s}\right\}\right] d s .
\end{aligned}
$$

Now, by taking $\lambda(s)=K \theta(s)$, we find a lower bound of $\mathfrak{z}_{T}$ on the set $\mathfrak{A}_{\delta}:=\left\{\sup _{t \leq T} \mid x_{t}^{K}-\right.$ $\left.u_{t} \mid \leq \delta\right\}$,

$$
\begin{aligned}
\log \mathfrak{z}_{T} \geq & K \int_{0}^{T}\left[\theta(s)\left(\dot{u}_{s}-\alpha u_{s}\right)-\frac{\sigma^{2}}{2} \theta^{2}(s) u_{s}\right] d s \\
& -\delta K\left[\left|\theta_{T}\right|+\int_{0}^{T}\left(\left|\dot{\theta}_{s}\right|+|\alpha \theta(s)|+\frac{\sigma^{2} \theta^{2}(s)}{2}\right) d s\right] .
\end{aligned}
$$

This lower bound jointly with (5.21) implies the following upper bound: for any absolutely continuous deterministic function $\theta(s)$,

$$
\varlimsup_{\delta \rightarrow 0} \varlimsup_{K \rightarrow \infty} \frac{1}{K} \log P\left(\mathfrak{A}_{\delta}\right) \leq-\int_{0}^{T}\left[\theta(s)\left(\dot{u}_{s}-\alpha u_{s}\right)-\frac{\sigma^{2}}{2} \theta^{2}(s) u_{s}\right] d s .
$$

Since $u_{s}$ is only nonnegative, it makes sense, for computational convenience, to use a corrected upper bound, with $\varepsilon>0$,

$$
\varlimsup_{\delta \rightarrow 0} \varlimsup_{K \rightarrow \infty} \frac{1}{K} \log P\left(\mathfrak{A}_{\delta}\right) \leq-\int_{0}^{T}\left[\theta(s)\left(\dot{u}_{s}-\alpha u_{s}\right)-\frac{\sigma^{2}}{2} \theta^{2}(s)\left(u_{s}+\varepsilon\right)\right] d s .
$$

If $\dot{u}_{t}$ is absolutely continuous function, a choice of $\theta(s)=\left(\dot{u}_{s}-\alpha u_{s}\right) / \sigma^{2}\left(u_{s}+\varepsilon\right)$ provides

$$
\begin{aligned}
\varlimsup_{\delta \rightarrow 0} \varlimsup_{K \rightarrow \infty} \frac{1}{K} \log P\left(\mathfrak{A}_{\delta}\right) & \leq-\frac{1}{2 \sigma^{2}} \int_{0}^{T} \frac{\left(\dot{u}_{s}-\alpha u_{s}\right)^{2}}{u_{s}+\varepsilon} d s \\
& \searrow-\frac{1}{2 \sigma^{2}} \int_{0}^{T} \frac{\left(\dot{u}_{s}-\alpha u_{s}\right)^{2}}{u_{s}} d s, \quad \varepsilon \backslash 0 .
\end{aligned}
$$


In general case, one can choose a sequence $\theta_{n}(s), n \geq 1$, of absolutely continuous functions such that

$$
\begin{aligned}
\lim _{n \rightarrow \infty} & {\left[\theta_{n}(s)\left(\dot{u}_{s}-\alpha u_{s}\right)-\frac{\sigma^{2}}{2} \theta_{n}^{2}(s)\left(u_{s}+\varepsilon\right)\right] } \\
& =\sup _{\phi \in \mathbb{R}}\left[\phi\left(\dot{u}_{s}-\alpha u_{s}\right)-\frac{\sigma^{2}}{2} \phi^{2}\left(u_{s}+\varepsilon\right)\right]=\frac{1}{2 \sigma^{2}} \frac{\left(\dot{u}_{s}-\alpha u_{s}\right)^{2}}{u_{s}+\varepsilon} .
\end{aligned}
$$

Hence, for sufficiently large $n,\left[\theta_{n}(s)\left(\dot{u}_{s}-\alpha u_{s} d s\right)-\left(\sigma^{2} / 2\right) \theta_{n}^{2}(s)\left(u_{s}+\varepsilon\right)\right] \geq 0$. Then, due to (5.25) being valid with $\theta(s)$ replaced by $\theta_{n}(s)$, and Fatou's theorem, we find that

$$
\begin{aligned}
\varlimsup_{\delta \rightarrow 0} \varlimsup_{K \rightarrow \infty} \frac{1}{K} \log P\left(\mathfrak{A}_{\delta}\right) & \leq-\varliminf_{n \rightarrow \infty} \int_{0}^{T}\left[\theta_{n}(s)\left(\dot{u}_{s}-\alpha u_{s}\right)-\frac{\sigma^{2}}{2} \theta_{n}^{2}(s)\left(u_{s}+\varepsilon\right)\right] d s \\
& \leq-\int_{0}^{T} \underline{\lim _{n \rightarrow \infty}}\left[\theta_{n}(s)\left(\dot{u}_{s}-\alpha u_{s}\right)-\frac{\sigma^{2}}{2} \theta_{n}^{2}(s)\left(u_{s}+\varepsilon\right)\right] d s \\
& =-\frac{1}{2 \sigma^{2}} \int_{0}^{T} \frac{\left(\dot{u}_{s}-\alpha u_{s}\right)^{2}}{u_{s}+\varepsilon} \searrow-\frac{1}{2 \sigma^{2}} \int_{0}^{T} \frac{\left(\dot{u}_{s}-\alpha u_{s}\right)^{2}}{u_{s}}, \varepsilon \searrow 0 .
\end{aligned}
$$

(iii) Verification. The proof of

$$
\varliminf_{\delta \rightarrow 0} \varliminf_{K \rightarrow \infty} \frac{1}{K} \log P\left(\sup _{t \leq T}\left|x_{s}^{K}-u_{s}\right| \leq \delta\right) \geq-\frac{1}{2 \sigma^{2}} \int_{0}^{T} \frac{\left(\dot{u}_{t}-\alpha u_{t}\right)^{2}}{u_{t}} d t
$$

is done in three steps.

(1) It suffices to analyze the case $\int_{0}^{T}\left(\left(\dot{u}_{s}-\alpha u_{s}\right)^{2} / u_{s}\right) d s<\infty$, which enables us to consider only those test functions that remain zero after arriving at zero. In other words, we will give the proof of (5.29) for absolutely continuous $u$. with $u_{0}=1$ and $\left(u_{t}>0\right)_{t<T}$, $u_{T} \geq 0$.

(2) Set $\tau_{C}=\inf \left\{t \leq T: x_{t}^{K} \geq C\right\}$, where $\inf \{\varnothing\}=\infty$ and notice that if for any $C>0$

$$
\varliminf_{\delta \rightarrow 0} \varliminf_{K \rightarrow \infty} \frac{1}{K} \log P\left(\sup _{t \leq T \wedge \tau_{C}}\left|x_{s}^{K}-u_{s}\right| \leq \delta\right) \geq-\frac{1}{2 \sigma^{2}} \int_{0}^{T} \frac{\left(\dot{u}_{t}-\alpha u_{t}\right)^{2}}{u_{t}} d t,
$$

then (5.29) holds. This can be seen as follows. Since

$$
\begin{aligned}
& \mathfrak{A}_{\delta} \supseteq\left\{\sup _{t \leq T \wedge \tau_{C}}\left|x_{t}^{K}-u_{t}\right| \leq \delta\right\} \cap\left\{\tau_{C}=\infty\right\} \\
&=\left\{\sup _{t \leq T \wedge \tau_{C}}\left|x_{t}^{K}-u_{t}\right| \leq \delta\right\} \backslash\left\{\sup _{t \leq T \wedge \tau_{C}}\left|x_{t}^{K}-u_{t}\right| \leq \delta\right\} \cap\left\{\tau_{C} \leq T\right\},
\end{aligned}
$$

we have $\left\{\tau_{C} \leq T\right\} \cup \mathfrak{A}_{\delta} \supseteq\left\{\sup _{t \leq T \wedge \tau_{C}}\left|x_{t}^{K}-u_{t}\right| \leq \delta\right\}$, so that

$$
2\left[P\left(\mathfrak{A}_{\delta}\right) \vee P\left(\tau_{C} \leq T\right)\right] \geq P\left(\sup _{t \leq T \wedge \tau_{C}}\left|x_{s}^{K}-u_{s}\right| \leq \delta\right) .
$$


Hence, due to (5.30),

$$
\begin{gathered}
\frac{\lim }{\delta \rightarrow 0} \frac{\lim }{K \rightarrow \infty} \frac{1}{K} \log P\left(\mathfrak{A}_{\delta}\right) \bigvee \lim _{C \rightarrow \infty} \frac{\lim }{K \rightarrow \infty} \frac{1}{K} \log P\left(\tau_{C} \leq T\right) \\
\geq-\frac{1}{2 \sigma^{2}} \int_{0}^{T} \frac{\left(\dot{u}_{t}-\alpha u_{t}\right)^{2}}{u_{t}} d t
\end{gathered}
$$

and it is left to recall that $\left\{\tau_{C} \leq T\right\}=\left\{\sup _{t \leq T} x_{t}^{K} \geq C\right\}$ and to refer to (5.3).

(3) By (1), $\int_{0}^{T} \dot{u}_{t}^{2} d t<\infty$. We proceed with the verification of (5.30). Define a continuous martingale $M_{t}$ and its variation process $\langle M\rangle_{t}$ : with $\varepsilon>0$,

$$
M_{t}=\int_{0}^{t \wedge \tau_{C}} \sqrt{K} \frac{\dot{u}_{s}-\alpha x_{s}^{K}}{\sigma \sqrt{x_{s}^{K}+\varepsilon}} d B_{s}, \quad\langle M\rangle_{t}=\int_{0}^{t \wedge \tau_{C}} K \frac{\left(\dot{u}_{s}-\alpha x_{s}^{K}\right)^{2}}{\sigma^{2}\left(x_{s}^{K}+\varepsilon\right)} d s .
$$

By definition of $\tau_{C}$, we have $\langle M\rangle_{T} \leq\left(2 K / \sigma^{2} \varepsilon\right) \int_{0}^{T}\left(\dot{u}_{t}^{2}+\alpha^{2} C^{2}\right) d s<\infty$, so that the stochastic exponential $\left(\mathfrak{z}_{t}, \mathscr{F}_{t}^{B}, P\right)_{t \leq T}$ with $\mathfrak{z}_{t}=e^{M_{t}-0.5\langle M\rangle_{t}}$ is a uniformly integrable martingale, $E_{\mathfrak{z}_{T}}=1$. We use the latter property to define a new probability measure $\bar{P}$ on $\left(\Omega, \mathscr{F}_{T}^{B}\right)$ by letting $d \bar{P}=\mathfrak{z}_{T} d P$ and applying

$$
P\left(\sup _{t \leq T \wedge \tau_{C}}\left|x_{t}^{K}-u_{t}\right| \leq \delta\right)=\int_{\left\{\sup _{t \leq T \wedge \tau_{C}}\left|x_{t}^{K}-u_{t}\right| \leq \delta\right\}} \mathfrak{z}_{T}^{-1} d \bar{P}
$$

for verification of (5.30). This approach heavily uses a semimartingale description of the processes $\left(x_{t}^{K}, \mathscr{F}_{t}^{B}, \bar{P}\right)_{t \leq T}$ and $\left(\mathfrak{z}_{t}^{-1}, \mathscr{F}_{t}^{B}, \bar{P}\right)_{t \leq T}$. We begin with the process $\left(B_{t}, \mathscr{F}_{t}^{B}, \bar{P}\right)_{t \leq T}$. The random processes $\left(B_{t}, \mathscr{F}_{t}^{B}, P\right)_{t \leq T}$ and $\left(\mathfrak{z}_{t}, \mathscr{F}_{t}^{B}, P\right)_{t \leq T}$ are continuous martingales and, in particular,

$$
d \mathfrak{z}_{t}=I_{\left\{\tau_{C} \geq t\right\}} \mathfrak{z}_{t} \sqrt{K} \frac{\dot{u}_{t}-\alpha x_{t}^{K}}{\sigma \sqrt{x_{t}^{K}+\varepsilon}} d B_{t} .
$$

Hence, the covariation process for $\mathfrak{z}_{t}, B_{t}$ is defined as

$$
\langle\mathfrak{z}, B\rangle_{t}=\int_{0}^{t \wedge \tau_{C}} \mathfrak{z}_{s} \sqrt{K} \frac{\dot{u}_{s}-\alpha x_{s}^{K}}{\sigma \sqrt{x_{s}^{K}+\varepsilon}} d s .
$$

It is well known (see, e.g., [17, Chapter 4 , Section 5]) that the random process $\left(\bar{B}_{t}, \mathscr{F}_{t}^{B}\right.$, $\bar{P})_{t \leq T}$ with

$$
\bar{B}_{t}=B_{t}-\int_{0}^{t} \mathfrak{z}_{s}^{-1} d\langle\mathfrak{z}, B\rangle_{s}=B_{t}-\int_{0}^{t \wedge \tau_{C}} \sqrt{K} \frac{\dot{u}_{s}-\alpha x_{s}^{K}}{\sigma\left(x_{s}^{K}+\varepsilon\right)} d s
$$


is a Brownian motion. Consequently, we find that, $\bar{P}$-a.s.,

$$
\begin{gathered}
x_{t}^{K}=1+\int_{0}^{t} I_{\left\{\tau_{C} \geq s\right\}} \dot{u}_{s} d s+\int_{0}^{t} \alpha x_{s}^{K}\left[1-I_{\left\{\tau_{C} \geq s\right\}}\left(\frac{x_{s}^{K}}{x_{s}^{K}+\varepsilon}\right)^{0.5}\right] d s+\int_{0}^{t} \frac{\sigma}{\sqrt{K}} \sqrt{x_{s}^{K}} d \bar{B}_{s}, \\
\log z_{t}^{-1}=-\int_{0}^{t \wedge \tau_{C}} \sqrt{K} \frac{\dot{u}_{s}-\alpha x_{s}^{K}}{\sigma \sqrt{x_{s}^{K}+\varepsilon}} d \bar{B}_{s}-\frac{1}{2} \int_{0}^{t \wedge \tau_{C}} K \frac{\left(\dot{u}_{s}-\alpha x_{s}^{K}\right)^{2}}{\sigma^{2}\left(x_{s}^{K}+\varepsilon\right)} d s .
\end{gathered}
$$

Now, we evaluate from below the value $1 / K \log _{\mathfrak{z}}^{-1}$ on the set $\left\{\sup _{t \leq T \wedge \tau_{c}}\left|x_{t}^{K}-u_{t}\right|\right\}$. Write

$$
\begin{aligned}
\frac{1}{K} \log \mathfrak{z}_{T}^{-1} \geq & -\frac{1}{2 \sigma^{2}} \int_{0}^{T} \frac{\left(\dot{u}_{s}-\alpha u_{s}\right)^{2}}{u_{s}} d s+h(C, \varepsilon, \delta) \\
& -\frac{1}{\sqrt{K}} \sup _{t \leq T}\left|\int_{0}^{t \wedge \tau_{C}} \frac{\dot{u}_{s}-\alpha x_{s}^{K}}{\sigma \sqrt{x_{s}^{K}+\varepsilon}} d \bar{B}_{s}\right|,
\end{aligned}
$$

where $h(C, \varepsilon, \delta) \underset{\delta \rightarrow 0}{\rightarrow} 0$. Therefore, (5.39) can be transformed into (here $\eta$ is a positive constant)

$$
\begin{aligned}
& \frac{1}{K} \log P\left(\sup _{t \leq T \wedge \tau_{C}}\left|x_{t}^{K}-u_{t}\right| \leq \delta\right) \\
& \geq-\frac{1}{2 \sigma^{2}} \int_{0}^{T} \frac{\left(\dot{u}_{s}-\alpha u_{s}\right)^{2}}{u_{s}} d s+h(C, \varepsilon, \delta) \\
&+\frac{1}{K} \log \bar{P}\left(\sup _{t \leq T \wedge \tau_{C}}\left|x_{t}^{K}-u_{t}\right| \leq \delta, \frac{1}{\sqrt{K}} \sup _{t \leq T}\left|\int_{0}^{t \wedge \tau_{C}} \frac{\dot{u}_{s}-\alpha x_{s}^{K}}{\sigma \sqrt{x_{s}^{K}+\varepsilon}} d \bar{B}_{s} \leq \eta\right|\right) .
\end{aligned}
$$

This lower bound makes it possible to claim that (5.30) holds true, provided that

$$
\begin{gathered}
\lim _{K \rightarrow \infty} \bar{P}\left(\sup _{t \leq T}\left|\int_{0}^{t \wedge \tau_{C}} \frac{\dot{u}_{s}-\alpha x_{s}^{K}}{\sigma \sqrt{x_{s}^{K}+\varepsilon}} d \bar{B}_{s}\right|>\sqrt{K} \eta\right)=0, \\
\lim _{\varepsilon \rightarrow 0} \varlimsup_{K \rightarrow \infty} \bar{P}\left(\sup _{t \leq T \wedge \tau_{C}}\left|x_{t}^{K}-u_{t}\right|>\delta\right)=0 .
\end{gathered}
$$

Since $I_{\left\{\tau_{s} \geq t\right\}}\left(\dot{u}_{s}-\alpha x_{s}^{K}\right)^{2} / \sigma^{2}\left(x_{s}^{K}+\varepsilon\right) \leq\left(\left|\dot{u}_{s}\right|+C\right)^{2} / \sigma^{2} \varepsilon$, the Doob inequality (here $\bar{E}$ is the expectation relative to $\bar{E}$ )

$$
\bar{E}\left(\sup _{t \leq T}\left|\int_{0}^{t \wedge \tau_{C}} \frac{\dot{u}_{s}-\alpha x_{s}^{K}}{\sigma \sqrt{x_{s}^{K}+\varepsilon}} d \bar{B}_{s}>\sqrt{K} \eta\right|\right) \leq \frac{4}{K \eta^{2}} \int_{0}^{T} \frac{\left(\left|\dot{u}_{s}\right|+C\right)^{2}}{\sigma^{2} \varepsilon} d s
$$

jointly with (3), establishes (5.42). 
Due to the first part of (5.39), the proof of (5.43) is reduced to the verification of

$$
\lim _{\varepsilon \rightarrow 0} \sup _{0 \leq x \leq C} x\left[1-\left(\frac{x}{x+\varepsilon}\right)^{0.5}\right]=0,
$$

which is obvious, and

$$
\lim _{K \rightarrow \infty} \bar{P}\left(\sup _{t \leq T}\left|\int_{0}^{t \wedge \tau_{C}} \sqrt{x_{s}^{K}} d \bar{B}_{s}>\sqrt{K} \eta\right|\right)=0,
$$

which is similar to the proof of (5.42).

\section{References}

[1] K. B. Athreya, Large deviation rates for branching processes. I. Single type case, The Annals of Applied Probability 4 (1994), no. 3, 779-790.

[2] K. B. Athreya and P. E. Ney, Branching Processes, Springer, New York, 2004.

[3] A. D. Barbour, Density dependent Markov population processes, Biological Growth and Spread (Proc. Conf., Heidelberg, 1979), Lecture Notes in Biomathematics, vol. 38, Springer, Berlin, 1980, pp. 36-49.

[4] J. D. Biggins and N. H. Bingham, Large deviations in the supercritical branching process, Advances in Applied Probability 25 (1993), no. 4, 757-772.

[5] C. Donati-Martin, A. Rouault, M. Yor, and M. Zani, Large deviations for squares of Bessel and Ornstein-Uhlenbeck processes, Probability Theory and Related Fields 129 (2004), no. 2, 261-289.

[6] M. I. Freidlin and A. D. Wentzell, Random Perturbations of Dynamical Systems, Fundamental Principles of Mathematical Sciences, vol. 260, Springer, New York, 1984.

[7] T. E. Harris, The Theory of Branching Processes, Die Grundlehren der Mathematischen Wissenschaften, vol. 119, Springer, Berlin; Prentice-Hall, New Jersey, 1963.

[8] Yu. Kifer, A discrete-time version of the Wentzell-Freidlin theory, The Annals of Probability 18 (1990), no. 4, 1676-1692.

[9] F. C. Klebaner, Population-dependent branching processes with a threshold, Stochastic Processes and Their Applications 46 (1993), no. 1, 115-127.

[10] - Introduction to Stochastic Calculus with Applications, 2nd ed., Imperial College Press, London, 2005.

[11] F. C. Klebaner and R. Liptser, Large deviations for recurrent sequences with aftereffect, Problemy Peredachi Informatsii 32 (1996), no. 4, 23-34.

[12] _ Asymptotic analysis and extinction in a stochastic Lotka-Volterra model, The Annals of Applied Probability 11 (2001), no. 4, 1263-1291.

[13] F. C. Klebaner and O. Nerman, Autoregressive approximation in branching processes with a threshold, Stochastic Processes and Their Applications 51 (1994), no. 1, 1-7.

[14] F. C. Klebaner and O. Zeitouni, The exit problem for a class of density-dependent branching systems, The Annals of Applied Probability 4 (1994), no. 4, 1188-1205.

[15] R. Liptser and A. A. Pukhalskii, Limit theorems on large deviations for semimartingales, Stochastics and Stochastics Reports 38 (1992), no. 4, 201-249.

[16] R. Liptser and A. N. Shiryayev, Statistics of Random Processes. II. Applications, Applications of Mathematics, vol. 6, Springer, New York, 1978.

[17] _ Theory of Martingales, Mathematics and Its Applications (Soviet Series), vol. 49, Kluwer Academic, Dordrecht, 1989.

[18] P. E. Ney and A. N. Vidyashankar, Local limit theory and large deviations for supercritical branching processes, The Annals of Applied Probability 14 (2004), no. 3, 1135-1166. 
[19] A. Puhalskii, Large Deviations and Idempotent Probability, Chapman \& Hall/CRC Monographs and Surveys in Pure and Applied Mathematics, vol. 119, Chapman \& Hall/CRC, Florida, 2001.

[20] - On some degenerate large deviation problems, Electronic Journal of Probability 9 (2004), no. $28,862-886$.

[21] L. C. G. Rogers and D. Williams, Diffusions, Markov Processes, and Martingales. Vol. 2. Itô Calculus, John Wiley \& Sons, New York, 1990.

F. C. Klebaner: School of Mathematical Sciences, Monash University, Building 28M,

Clayton Campus, Victoria 3800, Australia

E-mail address: fima.klebaner@sci.monash.edu.au

R. Liptser: School of Mathematical Sciences, Monash University, Building 28M,

Clayton Campus, Victoria 3800, Australia

E-mail addresses: rliptser@sci.monash.edu.au; liptser@eng.tau.ac.il 


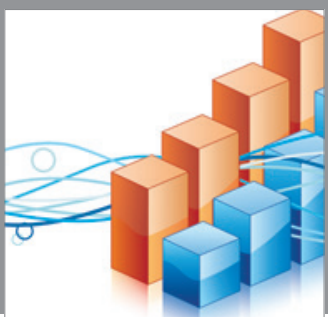

Advances in

Operations Research

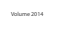

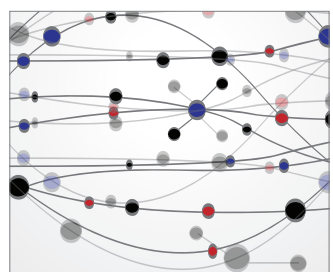

\section{The Scientific} World Journal
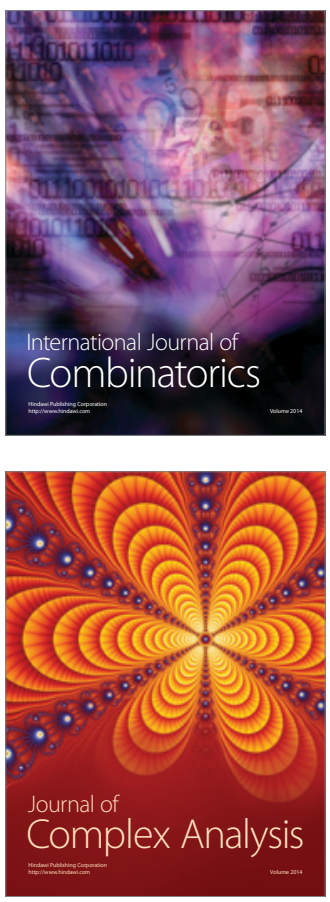

International Journal of

Mathematics and

Mathematical

Sciences
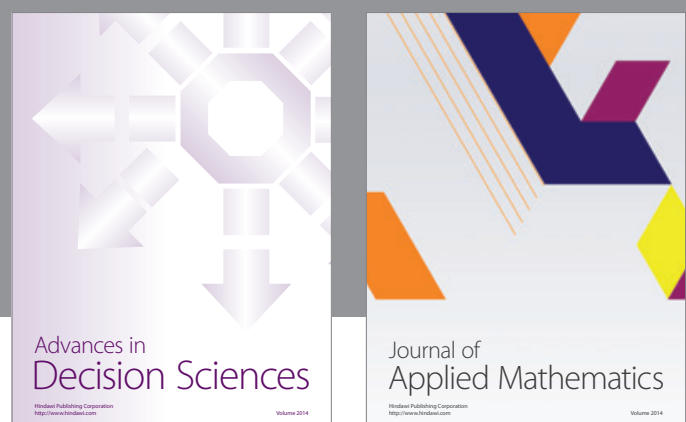

Journal of

Applied Mathematics
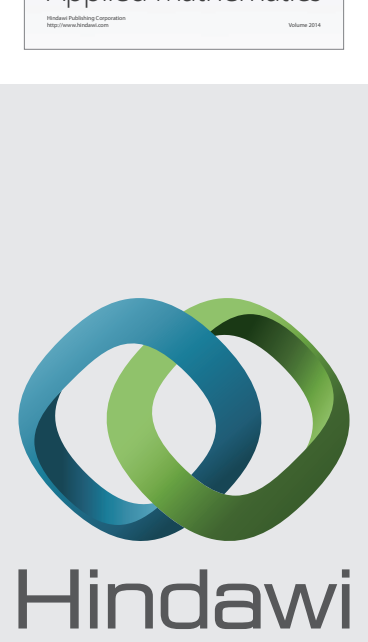

Submit your manuscripts at http://www.hindawi.com
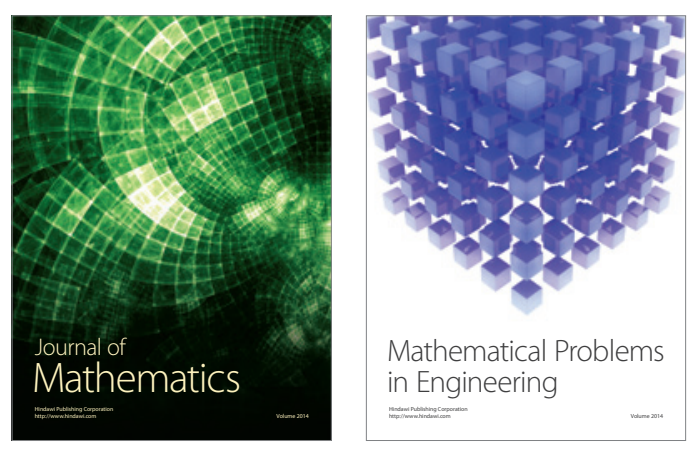

Mathematical Problems in Engineering
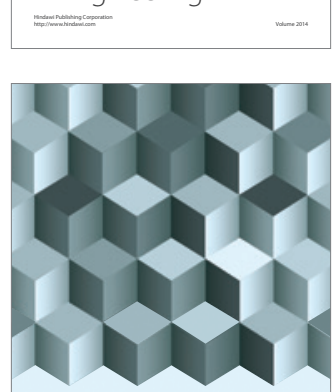

Journal of

Function Spaces
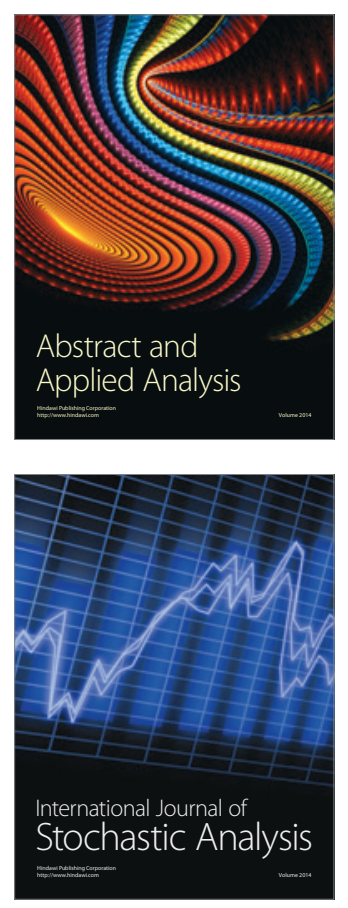

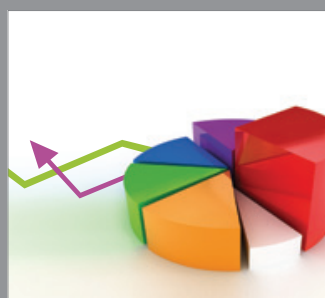

ournal of

Probability and Statistics

Promensencen
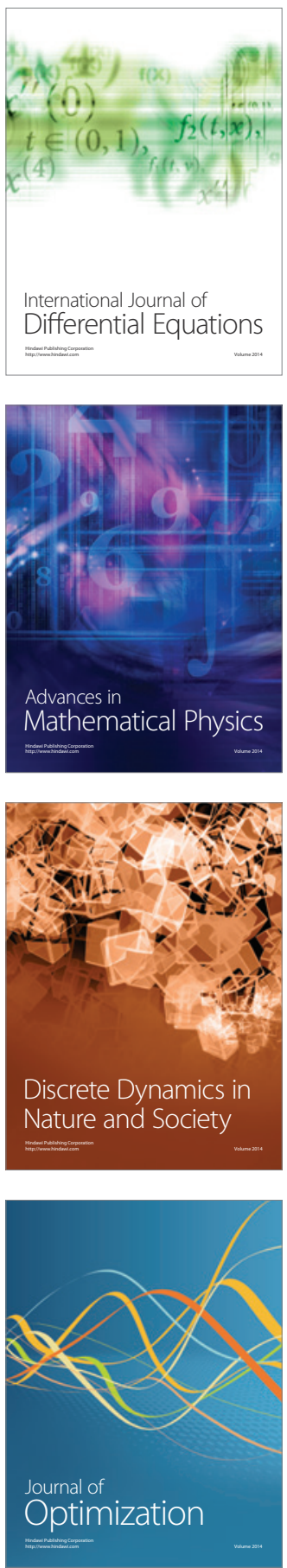